\title{
Region-Specific Automated Feedback in Temporal Bone Surgery Simulation
}

\author{
S Wijewickrema, I Ioannou, Y Zhou, P Piromchai, J Bailey, G Kennedy, S O'Leary
}

\begin{abstract}
The use of virtual reality simulators for surgical training has gained popularity in recent years, with an ever increasing body of evidence supporting the benefits and validity of simulation-based training. However, a crucial component of effective skill acquisition has not been adequately addressed, namely the provision of timely performance feedback. The utility of a surgical simulator is limited if it still requires the presence of experts to guide trainees. Automated feedback that emulates the advise provided by experts is necessary to facilitate independent learning. We propose an automated system that provides region-specific feedback on surgical technique within a temporal bone surgery simulator. The design of this system allows easy transfer of feedback models to multiple temporal bone specimens in the simulator. The system was validated by an expert otologist and was found to provide highly accurate and timely feedback.
\end{abstract}

Keywords-Automated Feedback in Surgery Simulation; Simulation-Based Surgical Training; Virtual Reality Temporal Bone Surgery;

\section{INTRODUCTION}

Computer-based simulation technologies such as virtual reality (VR) have been harnessed to develop cost-effective, risk-free platforms on which trainees can practice surgery at their own convenience, repeatedly if necessary. The popularity of VR surgical simulators has been aided by the use of technologies such as 3D stereoscopic rendering and haptic feedback [1], [2] which can be used to produce relatively realistic representations of real-life surgery .

One limitation in the practical use and wider adoption of VR simulation for surgical education is the requirement for the presence of expert trainers. The coaching and feedback provided by experts along with repeated practice are key ingredients in the acquisition of expertise [3]. Unfortunately the busy schedules of expert trainers limit the practice opportunities afforded to trainees, even when a simulator is available. The ideal solution to this problem is to embed automated real-time performance feedback within surgical simulators that emulates the advice provided by experts.

Previous work on the provision of real-time performance feedback in VR surgical simulators focussed on relatively simple forms of feedback. For example, in dental simulation, Rhienmora et al.[4] provided real-time feedback on individual metrics such as force, position, and orientation by making comparisons with average expert values. Fried et al.[5] introduced measures for evaluating performance in virtual endoscopic sinus surgery (such as violation of tissue, violation of instrument tolerances, force patterns etc.) and provided real-time feedback by making comparisons with a database of pre-recorded performances. In temporal bone surgery simulation, Sewell et al.[6] provided feedback on surgical technique based on individual metrics such as visibility, force, and region of bone removed. Kennedy et al.[7] used machine learning to provide automated feedback on the force applied by trainees when performing virtual temporal bone surgery.

All these approaches relied on relatively simple, univariate models for the provision of feedback. However, these types of feedback do not adequately consider the complex and multi-faceted nature of surgical expertise. Previous work suggests that there exist complex relationships between the various metrics that characterise surgical technique[8], [9], [10]. For example, some types of surgical technique may only be detected if we examine how two or more metrics vary together. Feedback based on individual metrics does not consider these relationships, nor does it closely emulate the meaningful and nuanced advice provided by human experts.

Another limitation of past approaches was a lack of context-awareness. A particular surgical technique may be desirable during one stage of a surgical procedure, but completely inappropriate during another stage. Therefore it is important to provide feedback that is cognisant of the technique that should be used in the given context.

Zhou et al.[9], [10] attempted to bridge this gap by dividing temporal bone surgery into a set of distinct stages and developing multi-variate machine learning models that define expert and trainee technique in each stage. Wijewickrema et al.[11] used these models to develop a feedback system that provides real-time advice on various aspects of surgical drilling technique. The system was shown to provide accurate and effective feedback [12].

Despite positive results, this system had some practical limitations. One problem was the requirement for accurate detection of surgical stage in order to provide relevant feedback. Surgeons do not perform the steps of a procedure in a strict sequential fashion, thus automated stage detection is a challenging problem in itself. Another limitation of stagebased feedback models is their lack of consideration for the unique anatomy of each temporal bone, which impedes their applicability to different temporal bone specimens.

In this paper, we expand on the work of Wijewickrema and Zhou et al. in an attempt to improve the accuracy and adaptability of the virtual temporal bone surgery feedback system. We eliminate the need for stage detection by proposing the use of region-based feedback models that reflect the distinct drilling techniques used when operating in different 
regions of the temporal bone. We present the results of a preliminary evaluation of the improved system conducted by an expert otologist.

\section{Virtual Reality Temporal Bone Surgery SIMULATOR}

The University of Melbourne VR temporal bone surgery simulator [11] was used as the platform for this work. The simulator displays three dimensional (3D) temporal bone models constructed from segmented MicroCT scans. The trainee interacts with the virtual bones using a haptic device, which acts as a surgical drill (figure 1).

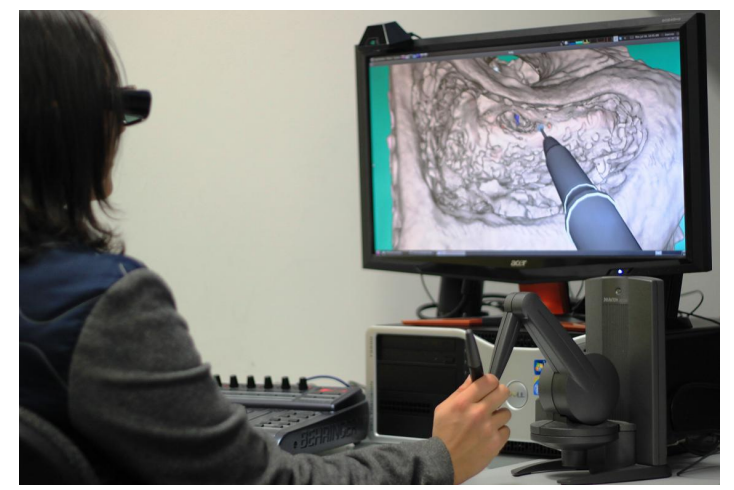

Figure 1. University of Melbourne VR temporal bone surgery simulator

The simulator allows practice of any otological drilling operation. During a procedure, the simulator records a variety of metrics (drill position, force, speed, material removal rate, distance to anatomical structures etc.) at a sample rate of approximately $15 \mathrm{~Hz}$. These recorded metrics can be used to replay the procedure in the simulator at a later time.

\section{Existing Feedback System}

The current system focusses on the provision of feedback to improve drilling technique, which is an important facet of otological surgical performance. To evaluate the quality of a trainee's technique, their actions need to be divided into smaller parts that can be analysed meaningfully. This is done by segmenting drilling trajectories into a series of strokes, where a stroke is a continuous drilling motion without significant change in direction. The end of a stroke is reached when drilling ceases, or when there is a change in direction. The simulator uses an automated algorithm to divide trajectories into strokes [11].

The quality of a trainee's surgical technique is evaluated by examining the features of their drilling strokes. The quality of each stroke is determined using properties such as its length, force, speed and proximity to anatomical structures. Certain simulator parameters such as burr size and magnification can also reflect good or bad drilling technique. Table I shows the metrics that were used to define surgical technique in the feedback system.

\begin{tabular}{|l|}
\hline Motion-Based Metrics \\
\hline Stroke Duration \\
Stroke Length \\
Stroke Speed \\
Stroke Acceleration \\
Stroke Force \\
Stroke Straightness \\
Average Distance to Centroid \\
\hline Proximity Metrics \\
\hline Stroke Distance to Anatomical Structures \\
\hline Simulator Parameters \\
\hline Average Zoom Level \\
Average Burr Size \\
\hline
\end{tabular}

Table I

STROKE METRICS USED TO DEFINE SURGICAL TECHNIQUE

Supervised learning techniques were used to classify expert and trainee behaviour based on the above stroke properties. In previous work, Zhou et al. developed and validated two such classification models (based on random forests and emerging patterns) [9], [10]. These models were trained using pre-recorded expert and trainee performances on the simulator. The procedure they performed involved the preparatory steps for cochlear implantation, namely cortical mastoidectomy followed by posterior tympanotomy and cochleostomy.

Different stages of this procedure require very different surgical technique, therefore the feedback system had to be context-aware. To achieve this, an expert otologist divided the procedure into sequential stages characterised by similar drilling technique. A separate classification model was trained for each stage, along with a classifier to detect the stage and choose the corresponding feedback model [10].

These classifiers are used in the feedback system to determine if and how a user's drilling technique differs to that of experts for the given stage, and to provide advise as to how they should modify their technique towards the expert gold standard [11]. In addition to the surgical technique feedback generated by the models, proximity warnings are also provided when the trainee approaches sensitive anatomical structures such as the facial nerve. Both surgical technique and proximity feedback are provided in the form of prerecorded verbal messages. Figure 2 illustrates the design of the automated feedback system.

\section{Limitations OF THE EXISTING System}

As discussed above, the existing feedback system uses separate classification models for each stage of the procedure. Therefore, it relies on timely and accurate stage detection to ensure the correct model is chosen at the correct time. Unfortunately, this is troublesome, as surgeons do not adhere to textbook procedures and tend to move back and forth between stages rather than completing them sequentially. This introduces noise in the form of inhomogeneous technique that impedes accurate classification. 


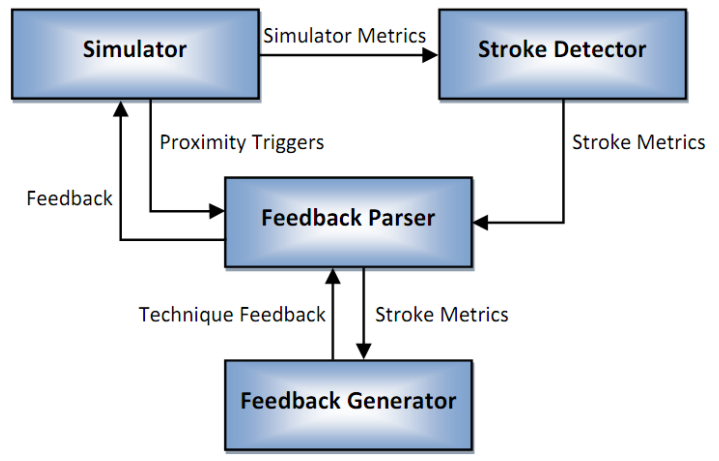

Figure 2. Design of the feedback system

Previous work [10] showed that stage prediction accuracy ranged from $81.38 \%$ to $93.46 \%$ depending on stage.

The issues introduced by real-time stage prediction are three-fold. First, less than ideal accuracy introduces errors into the system, as an inaccurate stage prediction leads to the selection of an inappropriate feedback model. Second, it adds an extra layer of computation to enable the detection of the current stage prior to the classification of technique and provision of feedback. Third, it does not consider the unique morphological features of each temporal bone, thus impeding the transferability of classification models to other virtual temporal bone specimens and necessitating the collection of new training data for each new specimen.

In addition to the issue of stage detection, further analysis of data collected in a previous validation study [11] showed that certain types of feedback provided by the system suffered from large inaccuracies. The majority of inaccurate feedback was in the form of zoom/magnification advice (table II). This indicates that the inclusion of simulation environment parameters in stroke technique feedback models may not be the best way to provide feedback on those aspects of performance.

\begin{tabular}{|l|r|}
\hline Feedback Type & Error Percentage \\
\hline Zoom Level & $61.54 \%$ \\
Stroke Length & $15.38 \%$ \\
Stroke Straightness & $13.46 \%$ \\
Stroke Speed & $5.77 \%$ \\
Force & $3.85 \%$ \\
\hline
\end{tabular}

Table II

BREAKDOWN OF INACCURATE FEEDBACK

Another shortcoming observed in the above validation study was that anatomical proximity alerts were relatively ineffectual at reducing damage caused to anatomical structures. A possible reason for this may be that proximity warnings alone are not informative enough to prevent damage. Instead, beginners may need to be told how they should use the drill near sensitive anatomical structures.

\section{Proposed Modifications}

In an effort to overcome the limitations identified in the existing automated feedback system, we propose modifications to: a) eliminate the need for real-time stage detection; b) enable the provision of more context-specific feedback; and c) improve accuracy by separating feedback on simulation parameters from surgical technique feedback.

\section{A. Removal of the need for real-time stage prediction}

To address the limitations imposed by stage prediction, it was necessary to devise an alternative method of segmenting the surgical procedure into sub-tasks with relatively homogeneous drilling technique. In the case of temporal bone surgery, it was observed that technique is not only dependent upon the stage of the procedure, but also on the region of the bone being drilled. In fact, the stages of the procedure largely correspond to the drilling of bone regions that are proximal to anatomical structures such as the dura, sigmoid sinus, incus, facial nerve, and round window. Therefore, we hypothesised that region-based feedback models based on proximity to these anatomical structures would be at least as effective as their stage-based equivalents.

We applied morphological operations [13] to segmented temporal bone MicroCT data to determine the regions near anatomical structures where distinct surgical technique is required. For example, to obtain the regions around anatomical structures (such as the dura and sigmoid sinus), dilation of the structure under consideration was performed along with a removal of non-bone voxels from the resulting area. To obtain regions between two or more anatomical structures (such as the facial recess region which is found between the facial nerve, chorda tympani and round window), we performed a dilation operation followed by erosion prior to the removal of non-bone voxels. Figure 3 shows the resulting regions. The accuracy of these regions was verified by two expert otologists.

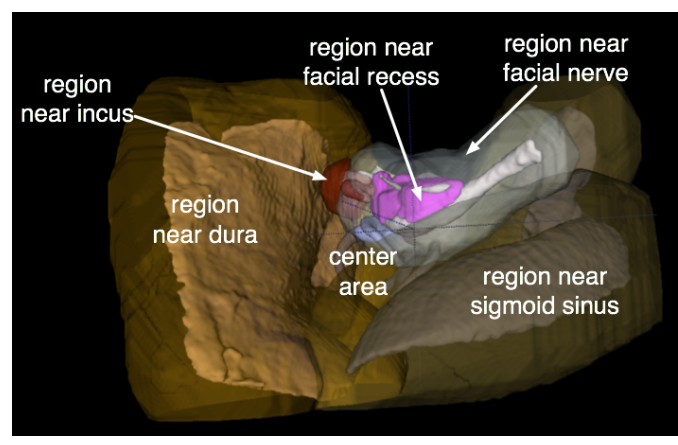

Figure 3. Regions of a temporal bone in which different stroke techniques should be used

Region calculations were performed offline and each voxel was mapped to its respective region. To create regionbased classifiers, we followed the same process outlined in 
Zhou et al.[9], [10] with the difference that strokes in the training data set were divided according to the region in which they occurred rather than their stage. The strokes in each region were used to train the corresponding classifier.

With this approach, the task of determining the region being drilled was reduced to an indexing operation. This not only decreased computational burden, but also eliminated the inaccuracies introduced by stage prediction. Further, the morphological operations used to define the regions can be easily adapted to new temporal bone specimens. Since regions are defined according to the unique morphology of each bone, surgical technique within each region is expected to be similar across different specimens. Therefore, the transferability of region-based classification models is also expected to be high.

\section{B. Presentation of context-specific feedback}

Real-time feedback in the existing system was provided in the form of pre-recorded messages (e.g. 'you could use more force', 'use longer stokes' etc.). These messages relied upon the trainee to understand the context in which the advise should be applied. With the introduction of regionbased feedback, it became possible to provide more accurate context-specific advise with region information (e.g. 'reduce force near the dura', 'use longer strokes near the facial nerve' etc.). The provision of such feedback complements anatomical proximity warnings and promotes a better understanding of the drilling technique that should be employed near sensitive organs to avoid unnecessary damage (e.g. the need to use less force near the facial nerve).

\section{Separation of simulator parameter feedback from the classification model}

Although metrics such as magnification level and burr size can be considered among the attributes that define good surgical technique, they can also be viewed as environment parameters that depend upon the region being drilled. Unlike stroke metrics which have a wide range of acceptable values, simulator parameters such as burr size and magnification have a narrow range of appropriate values in a given region, and these ranges can be defined accurately. Consequently, these metrics can be separated from the classification of stroke technique, and feedback can be provided using simple rule-based methods.

We employed the assistance of an otologist to define valid ranges for burr size and magnification level in each bone region. If a trainee consistently uses values outside these ranges, the system provides corrective feedback to bring the incorrect parameter within the acceptable range. When providing feedback, corrections to these environment variables are considered to be of higher priority than adjustments to stroke technique. For example, selection of a fitting burr size ensures efficient drilling in open areas and minimises damage in areas close to anatomical structures, while use of appropriate magnification affects the visibility and clarity of the operating space. Therefore, the feedback system was designed to allow for rule-based validation (and subsequent provision of feedback) of the environment parameters prior to the classification of strokes, as illustrated in figure 4.

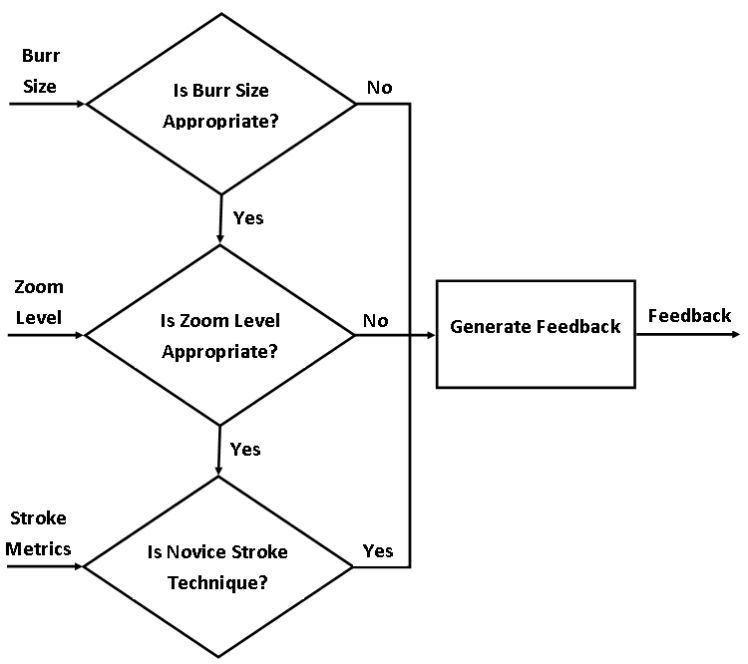

Figure 4. Design of the proposed feedback generator

\section{COMPlETE FEEDBACK SYSTEM WITH PROPOSED MODIFICATIONS}

The proposed changes were incorporated into the previously discussed feedback system [11]. The algorithm for the provision of feedback in the updated system is shown in algorithm 1 .

\section{VALIDATION}

We defined three error measures to determine the accuracy of the feedback generated by the system, as discussed in Wijewickrema et al.[11]:

1) false positives $(f p)$ : feedback was provided when stroke technique was acceptable,

2 ) wrong content $(w c)$ : stroke technique was accurately classified as unacceptable, but the feedback suggested the wrong adjustment, and

3) false negatives $(f n)$ : feedback was not provided when technique was unacceptable.

These error measures can be used to determine the timeliness and correctness of feedback. Timely feedback is the percentage of feedback provided when (and only when) improper technique is detected, and feedback accuracy is the percentage of correct feedback compared to the total feedback provided. Equations (1) and (2) show the definitions of timeliness and accuracy respectively, where $t f$ is the total feedback provided.

$$
\text { Timely Feedback }=\frac{t f-f p}{t f-f p+f n} \times 100 \%
$$


Input: $f b_{\text {trigger }}$ : feedback trigger, $s_{i}$ : new stroke

Output: $f b_{\text {pres }}$ : feedback to present define $n$ : required number of feedback repetitions $n_{r e p}$ : current number of feedback repetitions $f b_{\text {prev }}$ : previous feedback

$f b_{\text {curr }}$ : current feedback

$f b_{\text {tech }}$ : current technique feedback

$T$ : feedback repetition delay

set $f b_{\text {curr }}=N U L L$;

if $f b_{\text {trigger }}==$ proximity warning then

set $f b_{\text {curr }}=$ proximity feedback;

else

set $f b_{\text {tech }}=N U L L$;

if region changed then

set classifier;

set burr and zoom ranges;

set $n_{\text {rep }}=0$;

set $f b_{\text {prev }}=N U L L$;

end

if burr range violated then

set $f b_{\text {tech }}=$ burr size feedback;

else

if zoom level violated then

set $f b_{\text {tech }}=$ zoom feedback;

else

if $s_{i}$ is classified as trainee then

I generate $f b_{\text {tech }}=$ technique feedback; end

end

end

if $f b_{\text {tech }} \neq N U L L$ then

if $f b_{\text {prev }} \neq f b_{\text {tech }}$ then

set $f b_{\text {prev }}=f b_{\text {tech }}$;

set $n_{r e p}=0$;

else

set $n_{r e p}=n_{r e p}+1$;

end

end

if $n_{\text {rep }}==n$ then

set $f b_{\text {curr }}=f b_{\text {tech }}$;

set $n_{\text {rep }}=0$;

end

end

if $f b_{\text {curr }} \neq N U L L$ then

if $f b_{\text {curr }}==$ last presented feedback then

if time since last presented feedback $>T$ then

$f b_{\text {pres }}=f b_{\text {curr }}$;

end

else

$f b_{\text {pres }}=f b_{\text {curr }}$;

end

end

Algorithm 1: Region-specific feedback system

$$
\text { Accurate Feedback }=\frac{t f-f p-w c}{t f} \times 100 \%
$$

We evaluated the performance of the proposed automated feedback system against the above measures through an analysis conducted by an otologist. We used pre-recorded performances of twelve medical students who performed cortical mastoidectomy on the simulator without any automated feedback. The recorded performances were replayed in the simulator with automated feedback provided by region-specific classification models (random forests [9] and emerging patterns [10]). We elected to use pre-recorded performances in our evaluation to allow direct comparison of the random forest and emerging pattern classifiers on the same dataset. An otologist evaluated the feedback presented during the replayed performances according to the three criteria above (false positives, wrong content, and false negatives). The timeliness and accuracy of the feedback was determined using equations (1) and (2). Table III shows the results of this analysis.

\begin{tabular}{|l|r|r|}
\hline & Random Forests & Emerging Patterns \\
\hline Total Feedback & 379 & 508 \\
Timely Feedback & $86.24 \%$ & $92.00 \%$ \\
Accurate Feedback & $91.29 \%$ & $87.20 \%$ \\
\hline
\end{tabular}

Table III

ACCURACY AND TIMELINESS OF FEEDBACK PROVIDED BY REGION-BASED ClASSIFICATION MODELS

The two classification models were evaluated on the same surgical performances, so it was possible to compare the timeliness and accuracy of the feedback provided by the two models for each performance. A Kolmogorov-Smirnov test [14] indicated that the data did not comply with normality assumptions, so we employed the Kruskal-Wallis [14] test for these comparisons (with a significance level of 95\%). Neither the timeliness nor the accuracy of the feedback from the two region-based models showed significant differences ( $p=0.35$ and $p=0.28$ respectively).

A previous evaluation of the feedback system with stagebased random forest classifiers [12] showed that timely feedback was provided $88.6 \%$ of the time, and out of these feedback messages, $84.2 \%$ were accurate. The results shown in table III indicate that the emerging patterns region-based model outperformed the stage-based random forest model of the previous study in both timeliness and accuracy. A relative increase of $3.8 \%$ in timeliness and a relative increase of $3.6 \%$ in accuracy were observed. The region-specific random forest model exhibited the best accuracy of the three models, but the worst timeliness.

\section{DISCUSSION AND CONCLUSION}

The evaluation of the proposed region-specific feedback system yielded highly positive results. We showed that 
the timeliness and accuracy of automated feedback can indeed be improved by using region-specific models and separating drilling technique from environment parameters such as burr size and magnification level. Additionally, we showed that region-based classifiers based on random forests and emerging patterns are similarly capable of providing surgical technique feedback with relatively high accuracy and timeliness.

However, this analysis was conducted as a proof of concept and did not fully validate the effectiveness of the feedback system. Further evaluation through a user study is necessary, whereby trainees will be given feedback as they perform the surgical procedure. Such a study would determine whether the proposed context-specific feedback is effective in improving the surgical technique of trainees towards the expert ideal, and whether damage to anatomical structures can be reduced.

It should also be noted that although the feedback system developed for the entire cochlear implantation drilling procedure, the analysis conducted here examined only the first part of that procedure, namely cortical mastoidectomy. Further, the participants of the study comprised of medical students with no prior surgical experience, while the target group for this type of training is usually otology residents with some familiarity with temporal bone surgery. Future studies should encompass the latter stages of the cochlear implantation drilling procedure, as well as test the effects of automated feedback on the performance of otology residents. In addition, it would be beneficial to conduct further studies to test the effectiveness of the feedback system when adapted to multiple temporal bone specimens.

Despite the aforementioned limitations, the results of this study are promising and can be considered as a step in the path to the development of self-guided simulation-based surgical training systems. While our work has focussed on temporal bone surgery, the same region-based approach and algorithms may be used to develop automated feedback systems for other types of open surgery where surgical technique is dependent upon proximity to anatomical structures.

\section{ACKNOWLEDGMENT}

We would like to thank the Australian Research Council and Cochlear Ltd. for funding this research.

\section{REFERENCES}

[1] M. Agus, A. Giachetti, E. Gobbetti, G. Zanetti, and A. Zorcolo, "Real-time haptic and visual simulation of bone dissection," Presence, vol. 12, no. 1, pp. 110-122, 2003.

[2] J. Bryan, D. Stredney, G. Wiet, and D. Sessanna, "Virtual temporal bone dissection: a case study," in Proceedings of the Conference on Visualization. IEEE Computer Society, 2001, pp. 497-500.

[3] M. Cox, D. M. Irby, R. K. Reznick, and H. MacRae, "Teaching surgical skills: changes in the wind," New England Journal of Medicine, vol. 355, no. 25, pp. 2664-2669, 2006.
[4] P. Rhienmora, P. Haddawy, S. Suebnukarn, and M. N. Dailey, "Intelligent dental training simulator with objective skill assessment and feedback," Artificial Intelligence in Medicine, vol. 52, no. 2, pp. 115-121, 2011.

[5] M. Fried, R. Satava, S. Weghorst, A. Gallagher, C. Sasaki, D. Ross, M. Sinanan, J. Uribe, M. Zeltsan, H. Arora et al., "Identifying and reducing errors with surgical simulation," Quality and Safety in Health Care, vol. 13, no. suppl 1, pp. i19-i26, 2004.

[6] C. Sewell, D. Morris, N. H. Blevins, S. Dutta, S. Agrawal, F. Barbagli, and K. Salisbury, "Providing metrics and performance feedback in a surgical simulator," Computer Aided Surgery, vol. 13, no. 2, pp. 63-81, 2008.

[7] G. Kennedy, I. Ioannou, Y. Zhou, J. Bailey, and S. O'Leary, "Mining interactions in immersive learning environments for real-time student feedback," Australasian Journal of Educational Technology, vol. 29, no. 2, 2013.

[8] C. Sewell, D. Morris, N. H. Blevins, S. Agrawal, S. Dutta, F. Barbagli, and K. Salisbury, "Validating metrics for a mastoidectomy simulator," Studies in Health Technology and Informatics, vol. 125, p. 421, 2006.

[9] Y. Zhou, J. Bailey, I. Ioannou, S. Wijewickrema, G. Kennedy, and S. OLeary, "Constructive real time feedback for a temporal bone simulator," in Medical Image Computing and Computer-Assisted Intervention-MICCAI 2013. Springer, 2013, pp. 315-322.

[10] Y. Zhou, J. Bailey, I. Ioannou, S. N. Wijewickrema, S. O'Leary, and G. Kennedy, "Pattern-based real-time feedback for a temporal bone simulator." in VRST, 2013, pp. 7-16.

[11] S. Wijewickrema, I. Ioannou, Y. Zhou, P. Piromchai, J. Bailey, G. Kennedy, and S. O'Leary, "A temporal bone surgery simulator with real-time feedback for surgical training," Medicine Meets Virtual Reality 21: NextMed/MMVR21, vol. 196, p. 462, 2014

[12] S. Wijewickrema, P. Piromchai, Y. Zhou, I. Ioannou, J. Bailey, G. Kennedy, and S. O'Leary, "Developing effective automated feedback in temporal bone surgery simulation," Otolaryngology-Head and Neck Surgery, p. Accepted, 2015.

[13] R. M. Haralick, S. R. Sternberg, and X. Zhuang, "Image analysis using mathematical morphology," Pattern Analysis and Machine Intelligence, IEEE Transactions on, no. 4, pp. 532-550, 1987.

[14] G. W. Corder and D. I. Foreman, Nonparametric Statistics: A Step-by-step Approach. John Wiley \& Sons, 2014. 


\section{University Library}

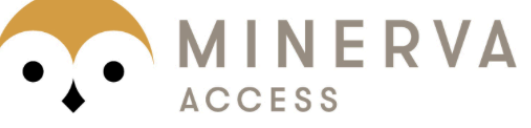

A gateway to Melbourne's research publications

Minerva Access is the Institutional Repository of The University of Melbourne

Author/s:

Wijewickrema, S;Ioannou, I;Zhou, Y;Piromchai, P;Bailey, J;Kennedy, G;O'Leary, S

Title:

Region-Specific Automated Feedback in Temporal Bone Surgery Simulation

Date:

2015

Citation:

Wijewickrema, S., Ioannou, I., Zhou, Y., Piromchai, P., Bailey, J., Kennedy, G. \& O'Leary, S. (2015). Region-Specific Automated Feedback in Temporal Bone Surgery Simulation. Traina, C (Ed.) Rodrigues, PP (Ed.) Kane, B (Ed.) Mazzoncini de Azevedo Marques, P (Ed.) Traina, AJM (Ed.) Computer-Based Medical Systems (CBMS), 2015 IEEE 28th International Symposium on, 2015-July, pp.310-315. IEEE. https://doi.org/10.1109/CBMS.2015.13.

Persistent Link:

http://hdl.handle.net/11343/282464 